\title{
Characterization of chromosomal and megaplasmid partitioning loci in Thermus thermophilus HB27
}

Haijuan Li, Angel Angelov, Vu Thuy Trang Pham, Benedikt Leis and Wolfgang Liebl*

\begin{abstract}
Background: In low-copy-number plasmids, the partitioning loci (par) act to ensure proper plasmid segregation and copy number maintenance in the daughter cells. In many bacterial species, par gene homologues are encoded on the chromosome, but their function is much less understood. In the two-replicon, polyploid genome of the hyperthermophilic bacterium Thermus thermophilus, both the chromosome and the megaplasmid encode par gene homologues (parABC and parABm, respectively). The mode of partitioning of the two replicons and the role of the two Par systems in the replication, segregation and maintenance of the genome copies are completely unknown in this organism.
\end{abstract}

Results: We generated a series of chromosomal and megaplasmid par mutants and sGFP reporter strains and analyzed them with respect to DNA segregation defects, genome copy number and replication origin localization. We show that the two ParB proteins specifically bind their cognate centromere-like sequences parS, and that both ParB-parS complexes localize at the cell poles. Deletion of the chromosomal parAB genes did not apparently affect the cell growth, the frequency of cells with aberrant nucleoids, or the chromosome and megaplasmid replication. In contrast, deletion of the megaplasmid parAB operon or of the parB gene was not possible, indicating essentiality of the megaplasmid-encoded Par system. A mutant expressing lower amounts of ParABm showed growth defects, a high frequency of cells with irregular nucleoids and a loss of a large portion of the megaplasmid. The truncated megaplasmid could not be partitioned appropriately, as interlinked megaplasmid molecules (catenenes) could be detected, and the ParBm-parSm complexes in this mutant lost their polar localization.

Conclusions: We show that in T. thermophilus the chromosomal par locus is not required for either the chromosomal or megaplasmid bulk DNA replication and segregation. In contrast, the megaplasmid Par system of $T$. thermophilus is needed for the proper replication and segregation of the megaplasmid, and is essential for its maintenance. The two Par sets in T. thermophilus appear to function in a replicon-specific manner. To our knowledge, this is the first analysis of Par systems in a polyploid bacterium.

Keywords: Partitioning genes (par), Thermus thermophilus, Chromosome, Megaplasmid, ParB

\section{Background}

All living cells have mechanisms ensuring the faithful segregation of the replicated genomes to the daughter cells. While the tubulin-based mitotic apparatus for DNA segregation used by eukaryotes is well studied, the mechanisms that mediate chromosome segregation in prokaryotic cells are less well understood. Evidence from

\footnotetext{
* Correspondence: wliebl@wzw.tum.de

Lehrstuhl für Mikrobiologie, Technische Universität München,

Emil-Ramann-Straße 4, D-85354 Freising-Weihenstephan, Germany
}

several groups demonstrates that bacterial chromosomes are also actively segregated and this segregation does not rely on cell growth [1-4]. Also, it has been shown that cytoskeletal proteins are also present in prokaryotic cells and they form mitotic-like apparatuses that provide force for active chromosome segregation $[5,6]$.

Several elements have been proposed which may make contributions to the dynamic movement of bacterial chromosomes [5,7]. For example, it has been suggested that DNA polymerase can provide force for bidirectional chromosome segregation in Bacillus subtilis cells $[8,9]$. 
Likewise, RNA polymerase has also been implicated to afford both incentive force and directionality for segregation through interacting with origin-proximal regions $[10,11]$. MreB is a chromosomally encoded actin homolog and in some rod-shaped bacteria it has been shown that MreB not only determines the cell shape, but is also involved in chromosome segregation [12-14].

Partitioning (par) genes have been known for a long time to play a pivotal role in the maintenance of lowcopy-number plasmids. Plasmid par locus usually contain three components: two ORFs encoding an ATPase (ParA) and a DNA-binding protein (ParB), and a centromere-like specific DNA sequence (parS). ParB binds its corresponding parS sequence, forming a large nucleoprotein complex. Low-copy-number plasmids with disrupted par loci localize improperly and are thus readily eliminated from host cells $[15,16]$. The molecular mechanisms by which par loci segregate plasmids have been studied to some extent. It has been suggested that ParA can form filaments which interact with ParB-parS complexes and provide force for segregation [17-20].

Many bacterial chromosomes encode orthologs of the plasmid partitioning proteins (Par) near their origin regions [21]. The first ParB-binding chromosomal parS sites were discovered in B. subtilis, where 10 pseudopalindromic 16 -bp sequences were identified in the $20 \%$ origin-proximal region of its chromosome. The presence of merely one such site could prevent the loss of an otherwise unstable plasmid from the host cell in a ParAB-dependent manner [22,23]. The consensus for the 16-bp sequence is $5^{\prime}$-TGTTNCACGTGAAACA-3'. Recently, this 16-bp sequence has been found in a large variety of bacteria, and in most cases, these sequences are origin-proximally located $[24,25]$. Normally, the corresponding par $A B$ genes can also be identified in such parS-containing chromosomes. While the crucial role of the par loci in plasmid partitioning has been well studied, their chromosomal counterparts are relatively poor investigated and the role of these Par systems in chromosome segregation is still disputable. The available data [26] support a model called diffusion ratchet mechanism where ParA uses the nucleoid as a matrix and ori-parS-ParB foci move by following a retracting ParA cloud.

Mutations introduced in chromosomal par loci usually have pleiotropic effects. In $B$. subtilis the chromosomal parAB orthologs are not essential genes, but they are involved in chromosome replication and segregation, chromosome origin localization and separation, and developmental gene regulation [27-29]. The parAB genes in Caulobacter crescentus on the other hand are essential and their depletion or overexpression results in defects in cell-cycle progression, cell division and chromosome segregation [30,31]. Each of the two chromosomes (chrI and chrII) of Vibrio cholerae contains a par locus (parABS1 and parABS2). It has been shown that parABS1 is probably involved in the segregation of the origin regions of chrI, but not of the bulk DNA of chrI or chrII [32,33]. In contrast, parABS2 can promote accurate subcellular localization and maintenance of the bulk DNA of chrII but not of chrI [34].

Although these diverse functions of chromosomal Par systems have been revealed to some extent in a few model organisms, the situation in other bacteria remains largely unknown, especially in bacteria containing more than one replicon. In addition to $V$. cholerae, there is only one study related to the par loci in bacteria possessing multiple replicons. Burkholderia cenocepacia has three chromosomes and a low-copy-number plasmid. Dubarry and co-workers [35] identified parS sites on the four replicons, and showed that the respective parABS systems are independent of each other.

Thermus thermophilus, which belongs to the phylogenetically deeply branching Deinococcus-Thermus phylum, has been established as a model organism for studying thermophilic bacteria. The genome of $T$. thermophilus consists of a chromosome $(1.89 \mathrm{Mb})$ and a megaplasmid $(0.23 \mathrm{Mb})$. It has been recently shown that $T$. thermophilus strains are polyploid and the chromosomal and megaplasmid copy number of the HB8 strain has been estimated to be four or five [36]. There are no reports in the literature regarding the chromosome and megaplasmid segregation in this organism, and the chromosome segregation mechanisms in polyploid bacteria have only recently begun to be addressed, e.g. in some polyploid cyanobacterial species $[37,38]$. In T. thermophilus, parAB gene homologues (termed here parABc and parABm, respectively) are also present both on the chromosome and on the megaplasmid [39]. Two studies have investigated the biochemical and structural properties of the chromosomal ParAc and ParBc proteins of T. thermophilus $[40,41]$. In this work, we address the functions of the chromosomal and megaplasmid par loci in T. thermophilus. We performed in vitro DNA binding assays with heterologously expressed ParB proteins, and generated a series of chromosomal and megaplasmid par mutants and sGFP reporter strains for subsequent analysis with respect to growth and DNA segregation defects, genome copy number and replication origin localization. The results from these experiments give first insights into how the two Par systems function in parallel in this thermophilic and polyploid bacterium.

\section{Results}

Genetic organization of the par loci in $T$. thermophilus

Both the chromosome and megaplasmid of T. thermophilus contain par loci [39], termed here parABc and 
parABm, respectively. The chromosomally encoded par locus consists of parAc (TT_C1605), parBc (TT_C1604) and parSc, organized in a way often found in bacterial chromosomes (Figure 1A). According to one report [42] and our own GC skew analysis (Additional file 1: Figure S1A), the chromosome replication origin (oriC) is positioned downstream of dnaA (TT_C1608), thus the parABc locus is located close to oriC ( $\sim 6 \mathrm{kbp}$ distance) (Figure 1A). The parSc site was identified using the 16-bp consensus sequence (5'-TGTTNCACGTGAAACA-3') allowing for one base pair mismatching and only one site was found [24]. When two or three mismatches were allowed, no other sequences could be found. The parSc sequence is located within a gene ( id B ) encoding $16 \mathrm{~S}$ rRNA methyltransferase immediately upstream of parAc (Figure 1A). Based on GC skew analysis, the cumulative minimum indicating the megaplasmid replication origin position was observed around the open reading frame of TT_P0079 (Additional file 1: Figure S1B; Figure 1A), thus the megaplasmid par $A B$ genes (TT_P0084 and TT_P0083) are also in the vicinity of the predicted megaplasmid oriC (Figure 1A). One 14-bp palindromic sequence (5'-AAGGACGCGTCCTT-3') was found in the parBm gene, and we provide evidence that this sequence could serve as the megaplasmid parS site (i.e. ParBm binding site, see below).

The organization of the $T$. thermophilus chromosomal par locus is similar to that of other bacterial chromosomal par systems [42]. The structure and genetic context of the parABm operon, on the other hand, is similar to the situation in low-copy-number plasmids where the par genes are adjacent to the repA gene for a plasmid-like replication initiator and are flanked by direct repeats. The chromosomal

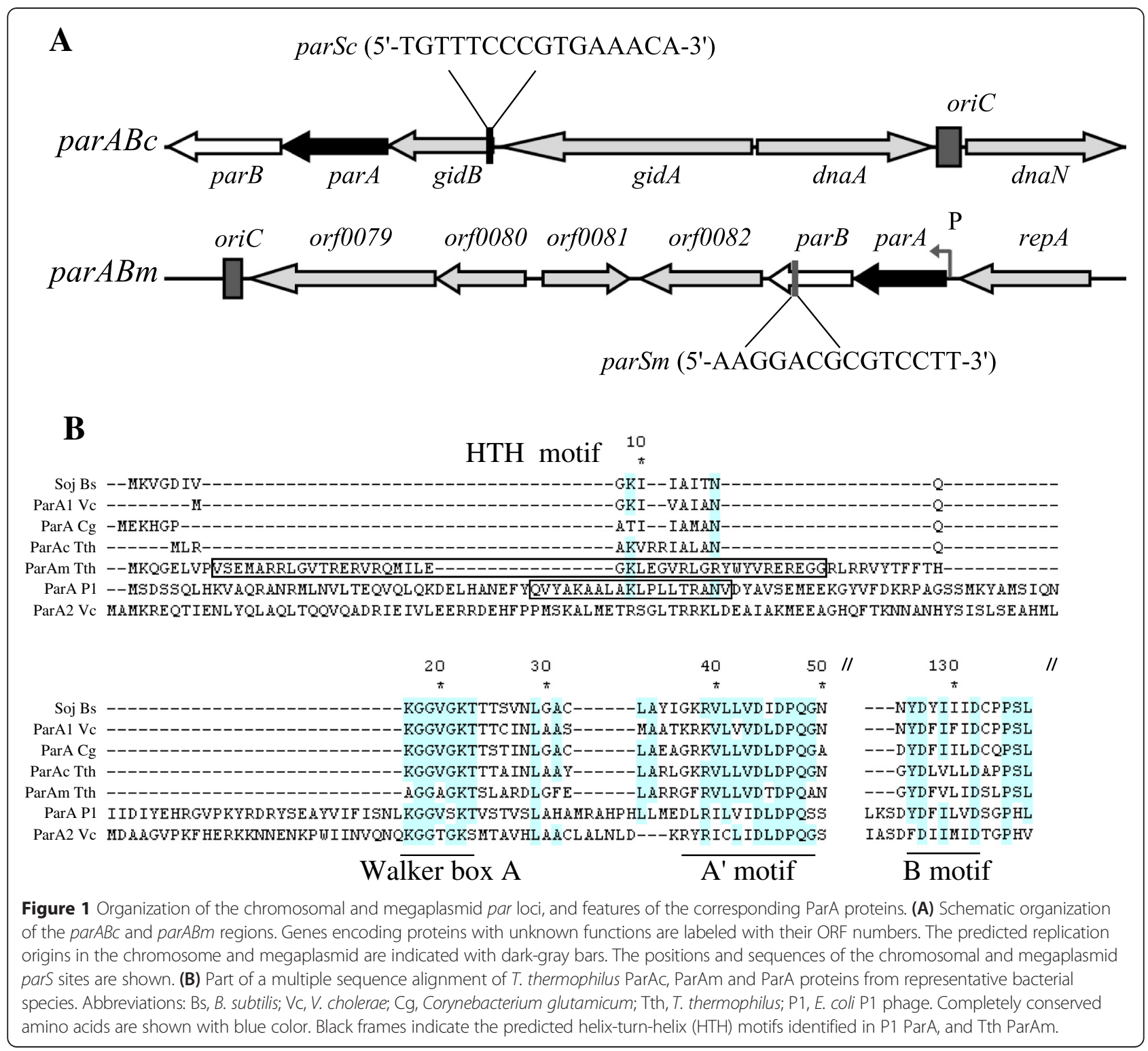


and megaplasmid Par proteins also possess different features. While both ParAc and ParAm are Walkertype ATPases which contain a conserved P-loop ATP binding motif, they differ in their sizes $(249$ aa for ParAc and 322 aa for ParAm) as well as in the presence of an N-terminal helix-turn-helix motif (HTH) in ParAm (Figure 1B), a feature that normally appears in plasmid ParAs but not in their chromosomal counterparts [21].

\section{Generation of par mutants in $T$. thermophilus}

We initiated the investigation of the two distinct par loci in $T$. thermophilus by attempting to generate deletion mutants. The chromosomal parAB genes were replaced by a thermostable kanamycin resistance gene cassette (kat). Southern blot analysis showed complete deletion of the parABc operon in the resulting mutant, $\triangle \operatorname{par} A B c$ (Figure 2A). In the same way, we initially tried to replace the whole parABm operon in the megaplasmid
A

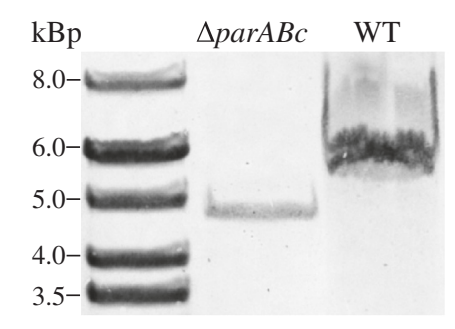

B

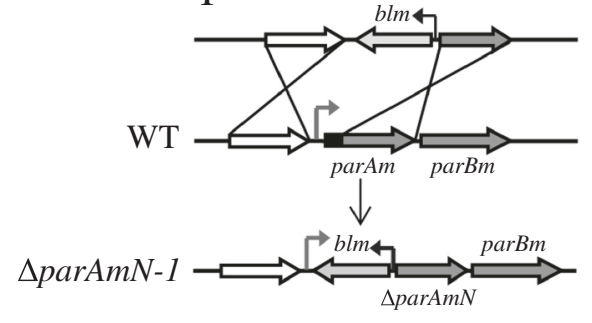

C

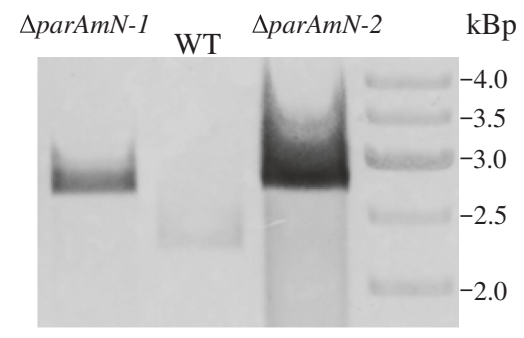

II

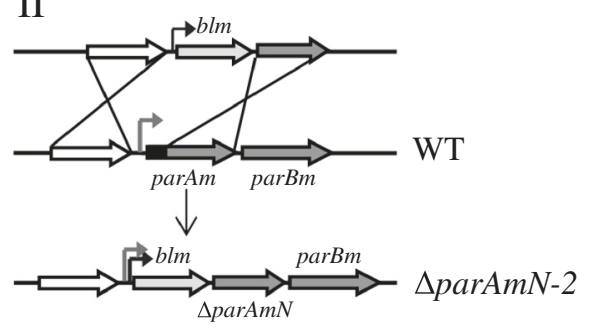

D

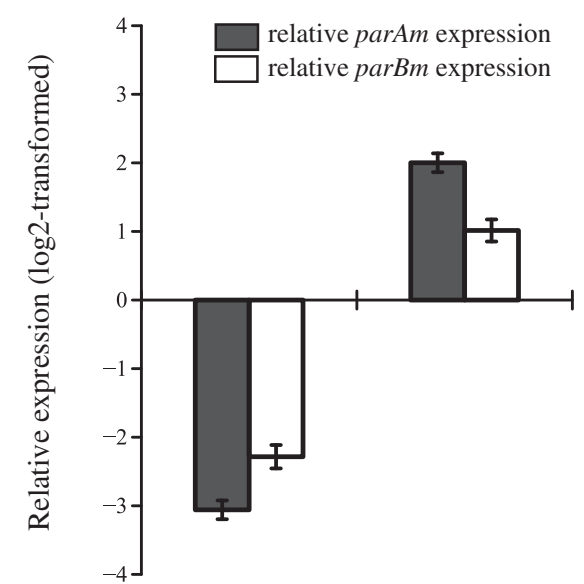

$\Delta$ parAmN-1 $\quad \Delta p a r A m N-2$

Figure 2 Generation and genotype confirmation of the chromosomal and megaplasmid par mutants. (A) Genotype confirmation of the parABc mutant by Southern blot. The genomic DNA was digested with BamHI and hybridization was performed with a 992-bp biotin-labeled DNA fragment. The in silico predicted sizes are $5.13 \mathrm{kbp}$ for the wild type and $4.68 \mathrm{kbp}$ for $\triangle p a r A B c$. (B) Schematic diagrams showing exchange of the N-terminus-encoding region of ParAm (amino acid positions 1-40) with blm. Gray arrowhead denotes the promoter region of parABm; black arrowhead, promoter of blm. (C) Genotype confirmation of the $\triangle p a r A m N-1$ and $\triangle p a r A m N-2$ mutants by Southern blot. The genomic DNA was digested with Pstl, the predicted sizes are $2.26 \mathrm{kbp}$ for the wild type and $2.73 \mathrm{kbp}$ for $\triangle p a r A m N-1$ and $\triangle p a r A m N-2$. (D) Transcription levels of the truncated parAm and parBm genes in $\triangle p a r A m N-1$ and $\triangle p a r A m N-2$ relative to those of the wild type determined by RT-qPCR. Gray bar represents the relative expression level of the truncated parAm, white bar represents that of parBm. The average values and SDs shown are from three experiments. 


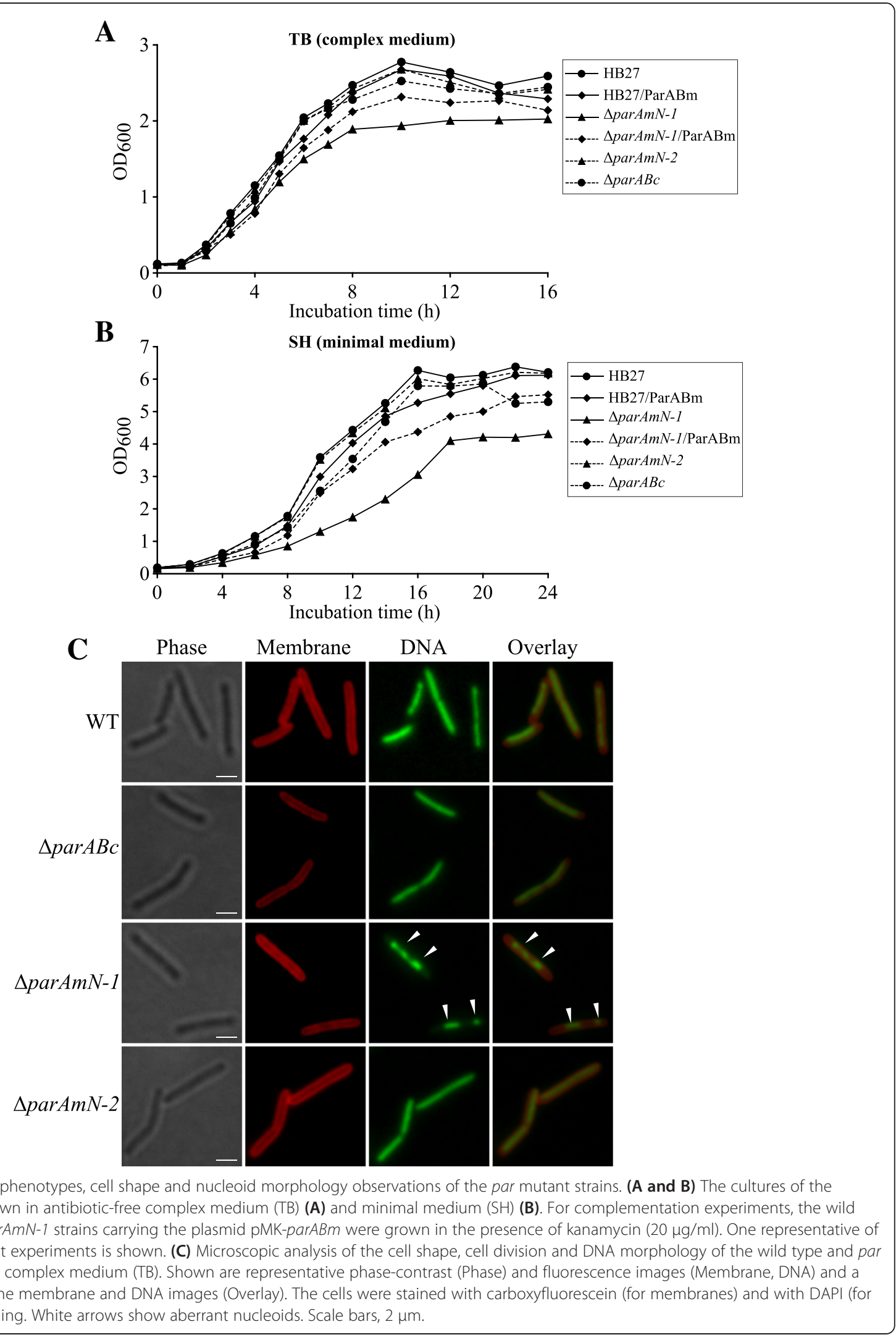


with a thermostable bleomycin resistance gene (blm). However, we were unable to obtain a null mutant: all the resulting transformants were found to be heterozygous, containing both the wild-type and the mutant alleles at the target locus (Additional file 2: Figure S2A). It is important to note that $T$. thermophilus is polyploid, and thus the heterozygous state is possible [36]. Thus, there appeared to be a strong selective pressure to retain parABm, i.e. parABm is probably essential. We then tried to knock down the parABm operon in order to test whether a perturbation in the amounts of ParAm and/or ParBm would yield a detectable phenotype, and also to pinpoint which of the two ORFs is essential. We constructed partial deletion variants of the parAm gene lacking the first 40 codons at the 5 '-end of the coding sequence but possessing the ribosome-binding sequence and start codon. Upstream of the new ORF start, the variants carried the $b l m$ marker with its strong promoter either in the opposite $(\triangle p a r A m N-1)$ or in the co-linear orientation $(\triangle p a r A m N-2)$ relative to the direction of parABm transcription (Figure 2B). Further, we also attempted to delete parBm by exchanging it with $b l m$. Complete exchange of parBm was not possible (Additional file 2: Figure S2B), while deletion of the N-terminusencoding sequences of the parAm was successful. Both anticipated deletion variants, which differed only in the orientation of the blm marker upstream of the truncated parAm ORF, were obtained as judged by Southern blot analysis (Figure $2 \mathrm{C}$ ). We then determined the transcription levels of the truncated parAm and parBm genes in the $\Delta$ parAmN-1 and $\triangle p a r A m N-2$ strains by RT-qPCR. As expected, in $\triangle p a r A m N-1$ the transcription levels of both genes were decreased: the relative mRNA amounts were 10-fold (for the truncated parAm) and 5-fold (for parBm) lower than that in the wild type, while in $\Delta$ par$A m N-2$ the levels of transcription of the two genes were 4-fold (for the truncated parAm) and 2-fold (for parBm) higher than that of the wild-type strain (Figure 2D).

\section{Growth rate and frequency of cells with aberrant nucleoids in the par mutants}

We analyzed the growth rates in complex (TB) and minimal $(\mathrm{SH})$ media of the par mutants. In both media, the $\triangle \operatorname{par} A B c$ strain did not display a considerable growth defect (Figure 3A and B). The $\triangle p a r A m N-1$ mutant grew more slowly and reached a lower final optical density in both media tested, while in $\triangle p a r A m N-2$, which differs from $\triangle$ parAmN-1 only by the direction of the resistance marker, these growth defects were not observed (Figure 3A and $\mathrm{B})$. The cell growth defect of $\triangle p a r A m N-1$ could also largely be complemented by introducing pMK-parABm and thereby providing a plasmid-borne copy of parABm (Figure $3 \mathrm{~A}$ and $\mathrm{B}$ ). Next, we performed microscopy experiments in order to examine cell morphology, DNA segregation and cell division of the $\triangle p a r A B c, \triangle \operatorname{par} A m N-1$ and $\triangle$ parAmN-2 mutants. When grown in complex medium, no cell morphology or cell division defects occurred in any of the mutants (Figure 3C). The frequencies of cells with aberrant nucleoids (as judged by the size and form of the DAPI-stained area) of $\triangle p a r A B c$ grown in either $\mathrm{TB}$ or $\mathrm{SH}$ medium were indistinguishable from those of the wild type (Table 1). Together with the growth phenotype (Figure $3 \mathrm{~A}$ and $\mathrm{B}$ ), these data suggested that deletion of par $A B C$ did not lead to appreciable changes in the genome bulk nucleoid replication or segregation. In the case of the $\triangle p a r A m N-2$ strain, the frequency of cells with irregular nucleoids was also not affected (Table 1), while in strain $\triangle p a r A m N-1$ an increased number of cells were found to contain apparently less and patchy-staining nucleic acid when grown in TB medium (Figure 3C; Table 1). These experiments showed that inadequate amounts of the megaplasmid ParAB proteins lead to genome segregation and/or replication defects and provoke defective cell growth.

\section{Genome content analysis of the par mutants}

To further understand the role of the Par proteins in genome maintenance, we analyzed the genome content of the par mutants using several methods. We inferred the relative amount of the megaplasmid by enzyme activity assays (for enzymes encoded on the megaplsmid, e.g. $\beta$-glucosidase encoded by ORF TT_P0042 and $\beta$-galactosidase encoded by TT_P0222) and we used quantitative PCR to measure the copy numbers per cell of both the megaplasmid and the chromosome. In line with the results of the growth and nucleoid visualization experiments (see above), which had indicated that par $A B c$ is neither involved in chromosome nor in megaplasmid bulk DNA replication and segregation, the replicon copy numbers in $\triangle p a r A B c$ did not differ from those of the wild type (Figure 4A,B and C; Table 1). In strain $\Delta$ parAmN-2, the chromosome copy number did not differ from that of the wild type also, while the value for the megaplasmid was mildly increased as judged from the enzyme activity assays and qPCR analysis (Figure 4A,B and C; Table 1). Consistent with this, PFGE analysis also showed that the chromosome and megaplasmid were intact in this mutant (Figure 4D). In

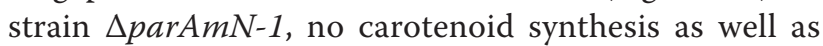
no $\beta$-glucosidase or $\beta$-galactosidase activity was detectable (Figure $4 \mathrm{~A}$ and $\mathrm{B}$ ), indicating that these megaplasmidencoded genes possibly were no longer present. Further qPCR, PCR and PFGE analyses showed that in strain $\Delta$ parAmN-1, the chromosomal DNA content appeared to be unchanged while a large portion of the megaplasmid was missing (Figure 4C,D,E and F; Table 1). The size of the resultant megaplasmid in this strain was 
Table 1 Frequencies of cells with aberrant nucleoids and relative genome copy numbers in par mutants and ParAm/ ParBm overexpression strains

\begin{tabular}{llllll}
\hline Strain & Cells with aberrant nucleoids (\%) & Relative TT_P0043 copies & Relative term copies & Relative oriCc copies & Relative terc copies \\
\hline WT & 1.24 & 1 & 1 & 1 & 1 \\
$\Delta$ parABC & 3.05 & $1.13 \pm 0.04$ & $1.09 \pm 0.05$ & $1.13 \pm 0.15$ & $1.14 \pm 0.08$ \\
$\Delta$ parAmN-1 & 33.02 & $/$ & $1.52 \pm 0.05$ & $0.91 \pm 0.13$ & $0.93 \pm 0.23$ \\
$\Delta$ parAmN-2 & 2.28 & $1.24 \pm 0.09$ & $1.26 \pm 0.16$ & $1.08 \pm 0.07$ & $1.12 \pm 0.02$ \\
TMP01 & 1.26 & $3.06 \pm 0.23$ & $2.45 \pm 0.19$ & $0.93 \pm 0.17$ & $0.85 \pm 0.12$ \\
TMP02 & 2.12 & $2.84 \pm 0.14$ & $2.29 \pm 0.35$ & $0.92 \pm 0.09$ & $0.89 \pm 0.07$ \\
TMP0 & 1.15 & $0.84 \pm 0.26$ & $0.95 \pm 0.05$ & $0.97 \pm 0.07$ & $1.05 \pm 0.03$ \\
\hline
\end{tabular}

The frequencies of cells with aberrant nucleoids were measured in cultures grown in TB medium; approximately 300 cells were analyzed for each strain. Relative genome copy numbers were determined by quantitative PCR. The mean values and the standard deviations of three independent experiments are shown. "/" indicates undetectable.

approximately $125-130 \mathrm{kbp}$ in contrast to $232.6 \mathrm{kbp}$ for the wild type (Figure 4D), and the coordinates of the eliminated region could be roughly mapped (Figure 4E). It seemed that this smaller megaplasmid could not be resolved properly after replication, as duplicated, triplicated, and even quadruplicated megaplasmid sizes could be observed (Figure 4D). Because the above effects were not present in strain $\triangle p a r A m N-2$, which differs from $\triangle \operatorname{par} A m N-1$ only by the orientation of the resistance marker and by the expression levels of the truncated ParAm and ParBm proteins (see above), we conclude that inadequate amounts of ParABm in $\triangle$ parAmN-1 led to the loss of large portions of the megaplasmid accompanied by megaplasmid resolution and segregation defects.

\section{Overexpression of ParAm and ParBm in T. thermophilus}

A slight but repeatedly detectable increase of the megaplasmid copy number was observed in the $\operatorname{parAmN-2}$ strain (Figure $4 \mathrm{~B}$ and $\mathrm{C}$; Table 1), which is characterized by a higher expression level of ParABm. For further clarification if the megaplasmid copy number is related to the amounts of ParAm and/or ParBm, we constructed two strains (TMP01 and TMP02) in which the parAm and $p a r B m$ genes were expressed from plasmids (pMKparAm and pMK-parBm, respectively). Both strains did not display obvious cell growth, cell morphology, cell division or DNA segregation defects. However, TMP01 and TMP02 were found to synthesize increased levels of carotenoids and displayed higher $\beta$-glucosidase activities (Figure 4A and B). Further, qPCR experiments demonstrated that both strains had 2.5 to 3.5 fold more megaplasmid copies compared to the control TMP0 strain (carrying the empty pMK18 vector), while the chromosomal copy number was unaffected (Figure 4C; Table 1). Moreover, entangled forms (catenenes) of the megaplasmids could be observed by PFGE analysis of TMP01 and TMP02, indicating that megaplasmid replication speed probably exceeded that of DNA separation and cell division (Figure 4D). Thus, it seems that both ParAm and ParBm act to promote megaplasmid replication.

\section{In vivo localization of the ParB proteins in T. thermophilus cells}

Studies of ParB localization patterns in other bacteria have shown that fusions of fluorescent proteins to ParB proteins form punctate fluorescent foci representing ParBparS nucleoprotein complexes in the cells [32,34,43]. To investigate the in vivo localization pattern of the T. thermophilus ParB proteins, we constructed C-terminal sGFP fusions of ParBc and ParBm. The sGFP variant used by us has been reported before [44], and it has been shown that it is able to fold and fluoresce properly when expressed in T. thermophilus growing at high temperatures (about $60^{\circ}$ C). When the ParBc-sGFP and ParBm-sGFP constructs were expressed in T. thermophilus TL-1 (a carotenoid synthesis deficient strain isolated in our group, permitting better observation of sGFP fluorescence), well-defined fluorescent foci could be observed (Figure 5A and F) providing in vivo evidence that the two ParB proteins can bind parS sites. Obviously, the polyploid nature of the cells is unproblematic with respect to distinct foci formation. In both cases, the majority of cells contained 2-6 foci with two of them localized at the cell poles ("old" poles), and the rest localized at positions of septum formation ("future" poles) (Figure 5A and F). For better illustration, the positions of the two most pole-proximal foci were measured from the nearest poles and expressed as fractions of the cell lengths. The plot of these measurements (approximately 120 cells were randomly selected for each strain) showed that the nearest-to-pole foci of ParBc-sGFP and ParBm-sGFP were on average in only $7.2 \%$ and $5.5 \%$ distance from the cell poles, indicating ParBc and ParBm are extremely polar localized (Figure 5E and I). Assuming that the subcellular locations of ParBc and ParBm actually also mark the positions of the corresponding parS regions and thus the respective chromosomal and megaplasmid origin regions (see Figure 1A), it 


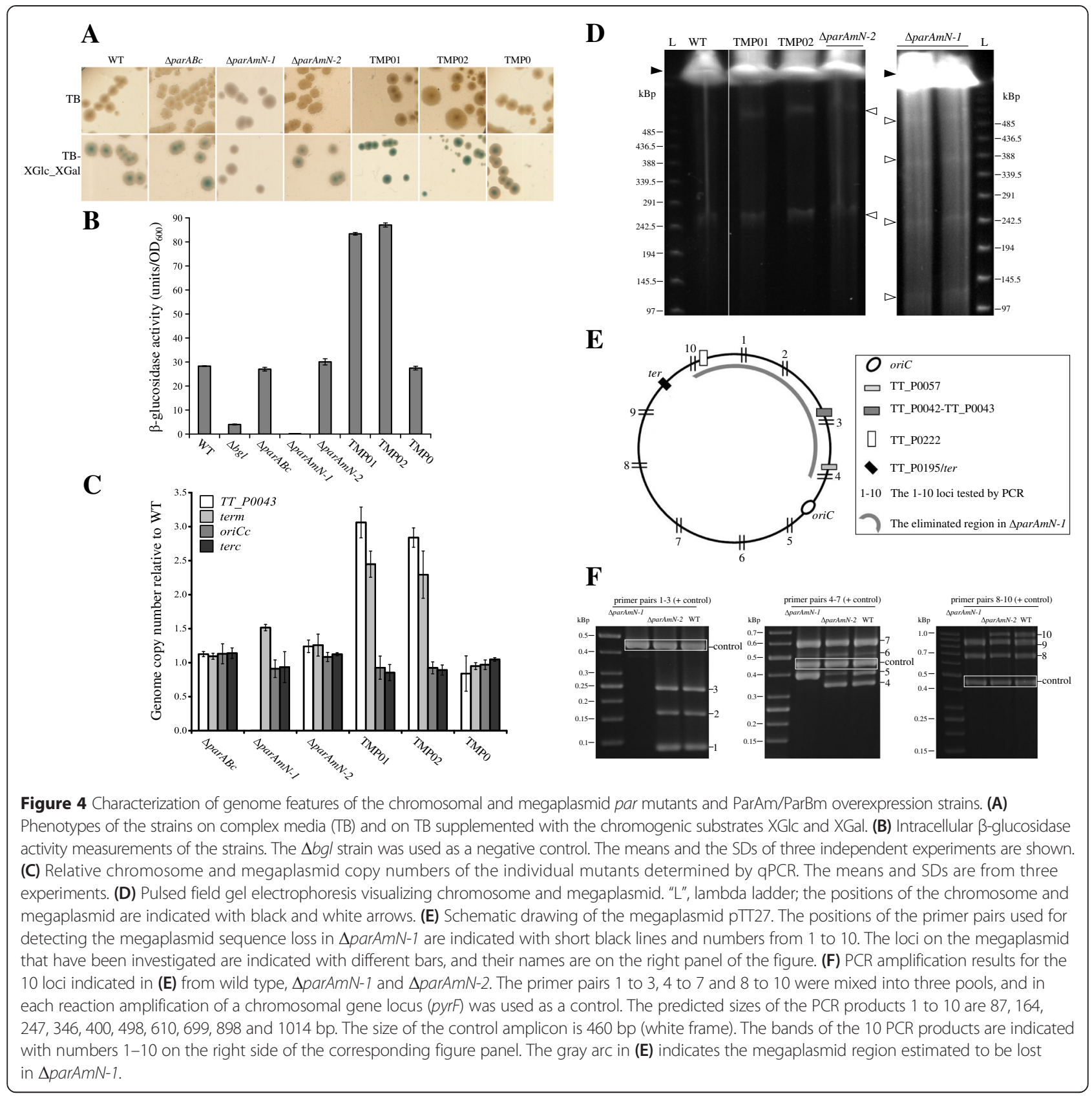

can be concluded that the origin regions of both replicons share a cellular localization near the cell poles. The fact that foci sometimes could also be detected at the cell centers or septum formation positions ("future" poles) indicated that ParB-parS (i.e., ParB-origin) nucleoprotein complexes might travel from cell poles to cell division positions, thus their cellular localization is dynamic.

As described above (Figure 4D), the $\Delta$ parAmN-1 strain lacking adequate ParABm amounts displayed megaplasmid segregation defects. We tested if also the subcellular location of ParBm (i.e. the subcellular location of parSm) was altered in this strain by expressing the ParBm-sGFP fusion in the $\triangle p a r A m N-1$ background. ParBm-sGFP also formed discrete foci in this strain (Figure 5G). However, the foci were mostly dissociated from the cell poles (Figure $5 \mathrm{G}$ ), i.e. most of the cells contained randomly positioned fluorescent foci. The average pole-proximal focus position (measured from the nearest poles) in cells that contained one focus was drastically increased compared with that in the wild-type cells (Figure 5J). This experiment showed that the decrease of the ParABm amounts and especially that of the ParAm amount (see Figure 2D), caused by reduced parABm expression, led to mislocalization 


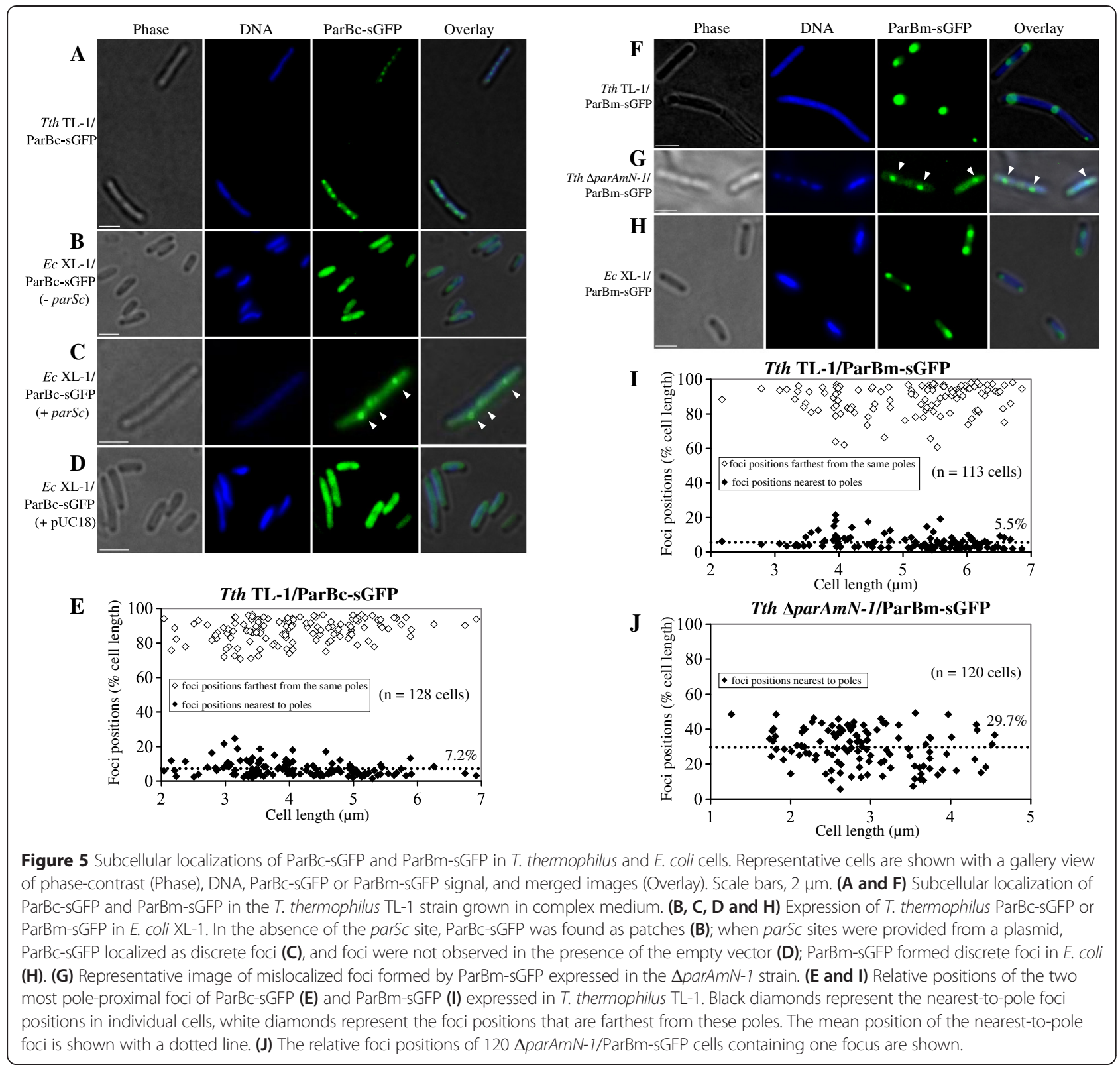

of the parSm sites and thus of the megaplasmid origin regions.

\section{In vivo localization of $T$. thermophilus $\mathrm{ParBc}$ and $\mathrm{ParBm}$ in}

\section{E. coli cells}

In order to better understand the factors that influence the ParB proteins' localization patterns, we expressed components of the $T$. thermophilus Par systems in $E$. coli, a host that does not encode chromosomal parABS system homologues. When T. thermophilus ParBcsGFP was expressed in E. coli, the fluorescence signal was spread over the nucleoid and no foci were formed (Figure 5B). Discrete fluorescent foci, which were randomly localized in the cells, could be observed only after the T. thermophilus parSc site was introduced into this strain (from a plasmid pUC- $\triangle$ parABc::kat) (Figure $5 \mathrm{C}$ ), and this effect was not observed in the empty vector control (pUC18) (Figure 5D). This means the ParBc subcellular localization pattern is dependent on the specific chromosomal parS site. On the contrary, we found that the megaplasmid ParB protein (expressed as ParBm-sGFP) formed foci when expressed alone in $E$. coli cells (Figure $5 \mathrm{H}$ ), suggesting that there were ParBm binding sites contributed by E.coli or by the parBm coding sequence itself. Since the 14-bp palindromic sequence could be readily identified in the parBm gene (see Figure 1A), we favored the latter option. In summary, the different localization patterns of ParBc and 
ParBm in E. coli cells suggested that the two ParBs bind different parS sites.

\section{In vitro DNA binding assays of the ParB proteins to parS sites}

The above experiments demonstrated that the parABc and parABm systems seem to play different cellular roles, and that the two ParBs also tend to only associate with their cognate parS sites. To further verify these observations, in vitro binding of recombinant ParB proteins to the 16-bp chromosomal parSc site and/or the predicted 14-bp megaplasmid parSm site were assayed by EMSA. These assays showed that ParBc could bind parSc and ParBm could bind the predicted parSm (Additional file 3: Figure 3SA and B). Almost all DNAbinding proteins contain more than one nucleic acid binding site, and during in vitro DNA binding assays they possibly bind any DNA non-specifically [45]. To test binding specificity, competition experiments with unlabeled probes were performed. In both cases, the unlabeled wild-type parSc/parSm probe competed much better than the unlabeled mutant parSc/parSm probe (Figure 6A and B). Thus the 16-bp parSc sequence and the 14-bp parSm sequence were bound specifically by ParBc and ParBm, respectively. Further, we performed EMSA of the ParB proteins with their non-cognate parS sites. These assays showed that ParBc did not bind specifically to parSm and ParBm did not bind specifically to parSc in vitro, as the respective mutant parS probe competed even better than the wild-type parS probe (Figure 6C and D). Thus, we conclude that the two ParB proteins bind parS sites in a replicon-specific manner.

\section{Discussion}

There are multiple copies of the chromosome and the megaplasmid in T. thermophilus [36] and whether their

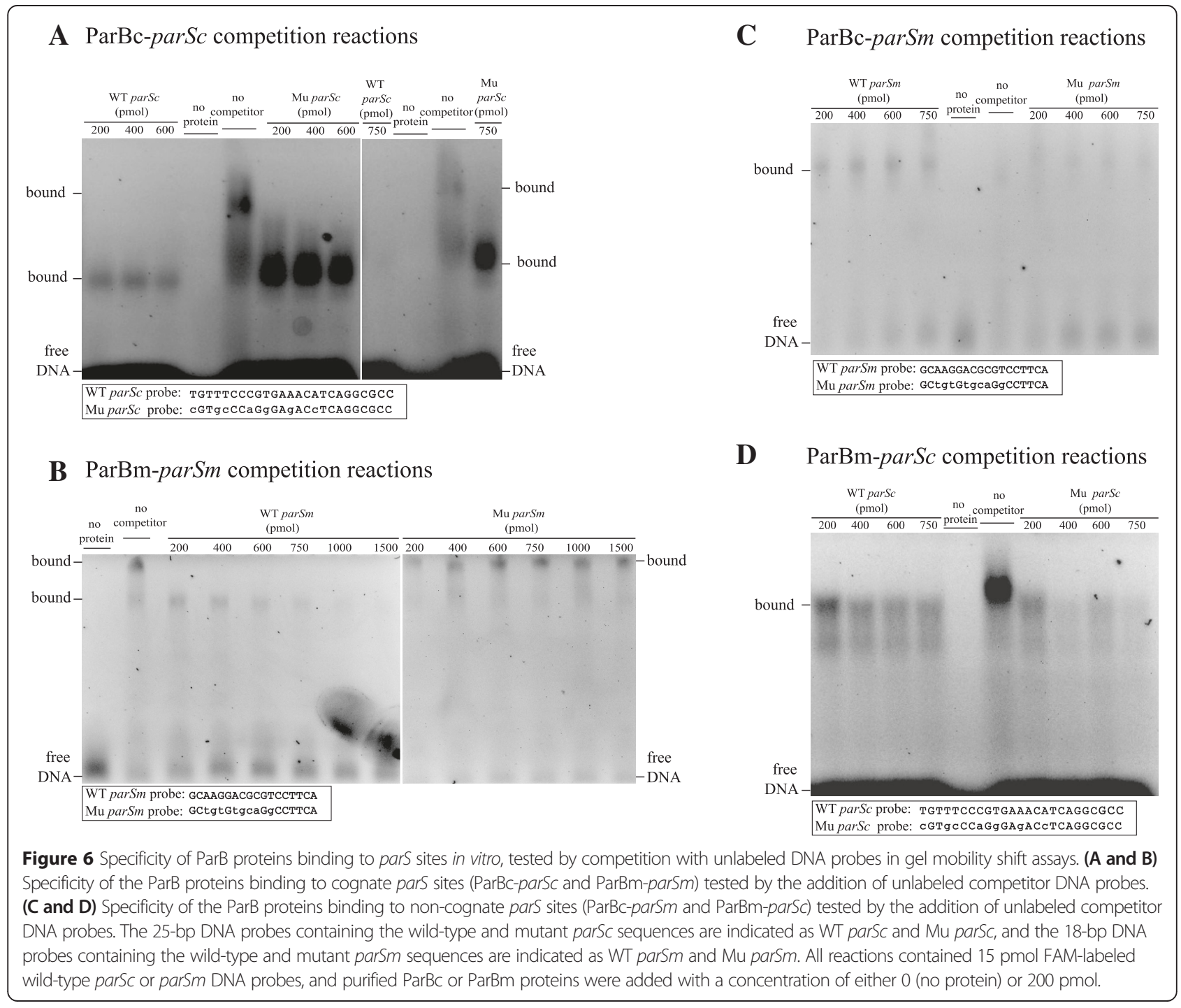


segregation is stringently regulated is not clear. Both the chromosome and the megaplasmid sequences of $T$. thermophilus strain HB27 revealed par loci. In this study, we investigate the characteristics and functions of the two par systems, thereby providing first insights in the mechanisms which may be involved in the genome partitioning in $T$. thermophilus.

\section{Characteristics of the chromosomal par locus}

Chromosomal Par orthologs seem to possess various functions in different bacterial species, and their role in chromosome segregation is suggested to be less pivotal compared with their counterparts in plasmids. Although deletion of spoOJ (parB) of B. subtilis leads to a considerable increase of anucleate cells during vegetative growth, the rest of the cells still exhibit a normal chromosome segregation pattern [27]; moreover, deletion of soj (parA) has no significant effect on chromosome segregation [46]. Similar observations have been made in some Gram-negative bacteria. In Pseudomonas putida, the parAB genes are not essential, and parA and parB mutations did not influence cell growth or chromosome segregation in rich medium [47]. In $V$. cholerae, deletion of parA1 does not alter cell growth and chromosome I (chrI) partitioning; however, the polar localization pattern of the origin region is abrogated, indicating that the parABS1 system functions to mediate the localization and segregation of the chrI origin region but not of the bulk nucleoid [32]. Our results show that in T. thermophilus the role of the chromosomal Par system is similar to that of the parABS1 system of $V$. cholerae. The parABc null mutant generated by us did not display apparent defects with respect to cell growth rate or frequency of cells with aberrant nucleoids (Figure 3; Table 1). Further observations from experiments targeting the copy numbers of the replicons showed that the chromosomal par locus was probably not required for either chromosome or megaplasmid bulk DNA replication and segregation (Figure 4A,B and C; Table 1). It is likely that the T. thermophilus chromosomal bulk nucleoid segregation is accomplished by other mechanisms. This conclusion is in line with the view that separate and redundant mechanisms may be involved to regulate the bacterial chromosome replication and segregation [48].

However, the chromosomal par locus may play other roles. Indeed, the in vitro DNA-binding assays showed that ParBc could bind the parSc site specifically (Additional file 3: Figure S3A; Figure 6A), indicating it is a functional ParB protein. In vivo, the ParBc-parSc complexes localized to the poles of wild-type $T$. thermophilus cells (Figure 5A and $\mathrm{E}$ ) and this localization was apparently dynamic, indicating that the origin regions were bound by $\mathrm{ParBc}$, and the nucleoprotein complexes were driven from "old" poles to "new" poles. It has been shown that in vitro the T. thermophilus ParAc can form dimers and then associate with DNA, forming nucleoprotein filaments, suggesting that ParAc has the capacity to mediate DNA movement [41]. Thus, similar to some other Par systems [25,32], the ParBc-origin complexes could possibly be anchored to the poles via ParAc filaments. Taken together, our data indicate that parABc is probably involved in the chromosomal origin region localization.

\section{Characteristics of the megaplasmid par locus}

The megaplasmid par locus is structured differently than the chromosomal par region. The parABm locus is also located in the megaplasmid origin-proximal region, and has a genetic set-up very similar to that found in some low-copy-number plasmids (Figure 1A). We could not obtain deletion mutants of the parABm operon or of the parBm gene, suggesting an essential role of parABm. Essentiality of par genes for bacterial cells has been observed for some chromosomal par systems. The null mutant of parB in C. cresentus is lethal [31], and direct deletion of the parAB2 genes in $V$. cholerae chromosome II is also not feasible [34]. To our knowledge, our work shows for the first time that a (mega)plasmid par locus is essential for its host organism. However, a parallel can be drawn from the case of the $V$. cholerae parAB2 locus on chromosome II, because this appears to be a megaplasmid-derived chromosome [49].

In the $T$. thermophilus $\triangle p a r A m N-1$ mutant, which expressed less ParABm, both the cell growth rate and the frequency of cells with irregular nucleoids were affected (Figure 3; Table 1). Furthermore, a substantial part (about $100 \mathrm{kbp}$ ) of the $232 \mathrm{kbp}$ megaplasmid, covering the region between approximately $11 \mathrm{kbp}$ and 111 $\mathrm{kbp}$ distance from one side of the megaplasmid origin, was lost in this mutant (Figure 4). This was not a spurious observation for just one clone, as all of 10 randomly selected $\triangle$ parAmN-1 colonies picked up directly from the transformation plates were found to have lost the same region of the megaplasmid. These phenotypes of the $\triangle p a r A m N-1$ strain were not observed in the isogenic mutant $\triangle$ parAmN-2 that differed from $\Delta$ par$A m N-1$ only by the direction of the antibiotic resistance cassette (and thus the transcription levels of the truncated parABm), suggesting that they were caused by inadequate amounts of ParABm in the $\triangle p a r A m N-1$ cells. Moreover, the truncated megaplasmid in $\triangle \operatorname{parAmN-1}$ seemed not to be decatenated properly, as multimeric forms of the megaplasmid could be observed by PFGE analysis (Figure 4D). In addition, the parSm sites (i.e. the megaplasmid origin regions) were dissociated from the cell poles and drastically mislocalized, as judged by the subcellular locations of the ParBm-sGFP fusion 
(Figure $5 \mathrm{G}$ and $\mathrm{J}$ ). These findings suggest that parABm probably mediates the accurate subcellular localization and segregation of the megaplasmid, resembling the function of the Par systems in most low-copy-number plasmids and some chromosomes of other bacteria.

The observation that only part of the megaplasmid was missing is in agreement with the conclusion that parBm is essential in T. thermophilus. It is likely that not only parBm but also other megaplasmid regions are essential and it seems that elimination of the entire megaplasmid is lethal to T. thermophilus. In support of this, we were not able to cure the megaplasmid from $T$. thermophilus despite various attempts (own unpublished work). The precise reason why the megaplasmid loss is not tolerated is currently unknown.

When we overexpressed either ParAm or ParBm in wild-type T. thermophilus cells (TMP01 and TMP02), the megaplasmid but not the chromosomal copy numbers were increased (Figure 4A,B,C and D; Table 1). This points to a role of parABm in megaplasmid replication initiation and/or copy number maintenance. It is possible that the $\mathrm{ParABm}$ proteins can activate the factors (e.g. RepA initiator) that are involved in the megaplasmid replication. A role of the ParA and ParB proteins in genome replication has been recently proposed also for other bacteria. In B. subtilis, Spo0J (ParB) was found to recruit a SMC condensin protein to replication origin regions, thereby promoting chromosome segregation [50,51]; the same phenomenon was also observed in Streptococcus pneumoniae [52]. ParB2 encoded by the $V$. cholerae chromosome II (chrII) was also found to influence the replication of chrII, in which ParB2 appeared to promote the replication by activating RctB protein that initiates chrII replication [53]. In the chromosome of $B$. subtilis and chromosome I of V. cholerae, ParA was found to directly interact with the chromosome replication initiator DnaA, thereby participating in the regulation of chromosome replication [54-56]. Taken together, it is conceivable that the ParABm system in T. thermophilus is important for maintaining the megaplasmid through regulating its replication and segregation. Interestingly, it also seems that segregation of the T. thermophilus megaplasmid is coordinated with its decatenation, which is reminiscent of the situations found in the E.coli or Streptomyces coelicolor chromosomes. Mutations of the parE gene (encoding one of the subunits of topoisomerase IV) in E.coli or S. coelicolor lead to chromosome catenation and fragmentation, thereby affecting the chromosome segregation $[57,58]$. It is possible that in the $T$. thermophilus $\triangle p a r A m N-1$ cells, the megaplasmid could not be decatenated properly due to the inadequate ParABm amounts, thus the megaplasmid was guillotined during separation into the daughter cells, and only those cells that recombined the essential portions of the megaplasmid would then survive. Apparently, further experiments (e.g. FISH) are needed in order to define whether the irregular nucleoid cells of the $\Delta$ par$A m N-1$ strain were cells that lacked the entire megaplasmid and thus were essentially dead cells or were cells that contained the chromosome and the "mini" megaplasmid.

\section{Chromosomal and megaplasmid Par are two independent systems}

In vitro, the $T$. thermophilus chromosomal ParBc and the megaplasmid ParBm could bind their corresponding parS site in a specific manner, and the Par proteins' binding to non-cognate parS sites was unspecific (Figure 6). These findings suggested that the two ParBs act only with their cognate parS sequences. This is supported by the in vivo ParB localization investigations in E. coli cells, as the two ParBs seemed to localize differently in this heterologous system (Figure 5B,C and $\mathrm{H}$ ). In E. coli cells, we found that ParBm-sGFP could form foci, and this further confirmed the conclusion drawn from the in vitro ParBm-parSm binding experiments, that is parBm contains its own binding site parSm.

The results of the in vitro ParB-parS bindings and of the in vivo ParB localization experiments, together with the fact that perturbation of the expression of parABm only affected the replication and/or segregation of the megaplasmid but not that of the chromosome, support the hypothesis that the two Par systems function independently. The phenomenon that $\operatorname{par} A B$ function in a replicon-specific manner has also been observed in other bacteria containing more than one Par system, for example the ParAB1 and ParAB2 systems of chromosomes I and II in $V$. cholerae [59] and the Par systems of the four replicons in B. cenocepacia [35]. From the bacteria with multiple replicons studied so far it seems like a common theme that their Par systems behave independently of each other rather than forming a network system with shared components.

\section{Conclusions}

One T. thermophilus cell contains multiple copies of the chromosome and megaplasmid. Like many bacteria, both the chromosome and the megaplasmid of $T$. thermophilus encode orthologs of the plasmid partitioning (par) genes, however their role in genome segregation is not known. In this study, we investigate the functions of these two Par systems in T. thermophilus through analysis of chromosomal and megaplasmid par gene mutants and ParAm/ParBm overexpression strains, as well as by using in vitro DNA binding assays of heterologously expressed ParB proteins and in vivo ParB protein localization observations. We show that in $T$. thermophilus the chromosomal ParAB system is not required for either the chromosomal or megaplasmid bulk DNA 
replication and segregation. It is however involved in the polar localization and separation of the chromosomal origin region. In contrast, the megaplasmid ParAB system in T. thermophilus probably functions to regulate the megaplasmid replication and segregation, thereby maintaining the megaplasmid. The two Par systems in T. thermophilus appear to function in a replicon-specific manner. Our study provides the first insights of the mode of operation of Par systems in a two-replicon, polyploid bacterium.

\section{Methods}

\section{Bacterial strains and growth conditions}

Escherichia coli XL-1 Blue (Agilent Technologies, Santa Clara, USA) was used as a host for DNA manipulations and was grown in LB medium (10 g/l tryptone, $5 \mathrm{~g} / \mathrm{l}$ yeast extract, $5 \mathrm{~g} / \mathrm{l} \mathrm{NaCl}$ ) at $37^{\circ} \mathrm{C}$. T. thermophilus $\mathrm{HB} 27$ (DSM 7039) and its derivative strains were grown at $60^{\circ} \mathrm{C}$ or $70^{\circ} \mathrm{C}$ with vigorous shaking in rich medium (TB) or nutritionally defined medium (SH). TB medium had a $\mathrm{pH}$ of 7.5 and contained per litre $8 \mathrm{~g}$ trypticase peptone, $4 \mathrm{~g}$ yeast extract, and $3 \mathrm{~g} \mathrm{NaCl}$, and was prepared with a high-carbonate mineral water (Purania, DRINKPOOL GmbH, Germany). SH medium was prepared as described in [60]. The growth media were supplemented with ampicillin $(100 \mu \mathrm{g} / \mathrm{ml}$ for E. coli), kanamycin $(20 \mu \mathrm{g} / \mathrm{ml}$ for E. coli and T. thermophilus $)$, bleomycin ("Bleocin", Calbiochem, $15 \mu \mathrm{g} / \mathrm{ml}$ ), chloramphenicol $(12.5 \mu \mathrm{g} / \mathrm{ml}$ for $E$. coli), XGlc (5-bromo-4chloro-3-indolyl- $\beta$-D-glucopyranoside, $50 \mu \mathrm{g} / \mathrm{ml}$ ) or XGal (5-bromo-4-chloro-3-indolyl- $\beta$-D-galactopyranoside, $50 \mu \mathrm{g} /$ $\mathrm{ml}$ ) when appropriate. All reagents were purchased from Sigma-Aldrich (Schnelldorf, Germany) except for growth media components which were obtained from BD Biosciences (Heidelberg, Germany).

\section{Strains and plasmids}

All strains and plasmids used are listed in Table 2. The oligonucleotides used are summarized in Additional file 4: Table S1. All allele exchange vectors (Table 2) for generating par mutants were derived from pUC18, and the constructs were obtained by Gibson assembly (New England Biolabs) [61]. In general, the upstream and downstream sequences (approximately $1 \mathrm{kbp}$ each) of the target regions, and the gene cassette encoding thermostable resistance to kanamycin (kat) or bleomycin $(b l m)$ (chemically synthesized using sequence data from [62]) were PCR-amplified using primers that generated sufficient overlaps. The purified PCR products of the two flanking regions and the kat/blm cassette were introduced into XbaI-digested pUC18 via four-fragment Gibson assembly reactions.

All the replicative vectors (Table 2) in T. thermophilus were derived from the E. coli/T. thermophilus shuttle vector pMK18 [63]. The constructs pMK-parAm, pMKparBm and pMK-parABm, which were generated by Gibson assembly as described above, carry parAm, parBm or the entire parABm operon, respectively, transcriptionally fused to kat of pMK18. In the same manner, the plasmid pMK-sgfp was obtained by adding the sgfp coding sequence, which was chemically synthesized using sequence data from [44], to kat of pMK18. The constructs pMKparBc-sgfp and pMKparBm$s g f p$ represent translationally fused $p a r B c$ and parBm to the $s g f p$ gene in pMK-sgfp. Codons encoding four glycine residues (poly-glycine linker) were introduced between parB and sgfp, and the ParB-sGFP fusions were expressed under the same promoter of the kat gene in pMK18.

The plasmids pET21a-parBc and pET21a-parBm were obtained by introducing purified parBc and parBm PCR fragments into XhoI, NdeI linearized pET21a by Gibson assembly.

\section{Quantitative PCR}

The quantitative PCR method for measuring the relative genome copies was performed as described in [64]. The chosen sites of the chromosome were near the origin (oriCc) and terminus (terc) regions, and those of the megaplasmid were the TT_P0043 locus (approximately $32 \mathrm{kbp}$ from the megaplasmid origin) and TT_P0195 locus (near the megaplasmid terminus (term)). Standard fragments used for quantification for each chosen locus were amplified by PCR using T. thermophilus genomic DNA as the template. The fragments were then purified from agarose gels and photometrically quantified. A series of dilutions containing defined numbers of the standard molecules were then used as templates for qPCR to generate standard curves. Cell extracts of the strains for qPCR were prepared by harvesting defined cell numbers (determined by spectrophotometry and with a Neubauer counting chamber) from exponentially growing cultures and resuspending in cell lysis buffer (Epicentre Biotechnologies, Germany); the cell lysis efficiency was determined by cell counting. After dialysis, dilutions were prepared from the cell lysates and aliquots were used as templates for qPCR. The sizes of the target amplicons were between 100 and $200 \mathrm{bp}$, and PCR was performed using qPCR Mastermix plus with fluorescein (Eurogentec, Germany) based on the protocol provided by the manufacturer. Three independent experiments were carried out for each strain. Standard curves were constructed from the $C_{T}$ values of the standard fragments and were later used to quantitate the genome copy numbers in the cell lysates.

\section{RT-qPCR}

For determining the relative expression levels of the truncated parAm and parBm genes, reverse transcription- 
Table 2 Strains and plasmids used in this study

\begin{tabular}{|c|c|c|}
\hline Name & Description & Source/reference \\
\hline \multicolumn{3}{|l|}{ Plasmids } \\
\hline $\mathrm{pUC18}$ & high-copy-number cloning vector & [68] \\
\hline pMK18 & E. coli/T. thermophilus shuttle vector, Tth (repA), Ec (oriE), $\mathrm{Km}^{\mathrm{R}}$ & [63] \\
\hline pUC- $\triangle$ parABc:.:kat & allele exchange vector for generating $\triangle p a r A B C$, ori $\mathrm{pUC}, \mathrm{Km}^{\mathrm{R}}$ & this study \\
\hline pUC- $\triangle$ parABm::blm & allele exchange vector for generating $\triangle p a r A B m$, ori $p \cup C, B \mid m^{R}$ & this study \\
\hline pUC- $\triangle$ parAmN-1 & allele exchange vector for generating $\triangle p a r A m N-1$, ori $p U C, B I m^{R}$ & this study \\
\hline pUC- $\triangle$ parAmN-2 & allele exchange vector for generating $\triangle p a r A m N-2$, ori $\mathrm{pUC}, \mathrm{BIm} \mathrm{m}^{\mathrm{R}}$ & this study \\
\hline pUC- $\Delta$ parBm::blm & allele exchange vector for generating $\triangle p a r B m$, ori $p U C, B I m^{R}$ & this study \\
\hline pMK-parAm & pMK18 derived vector, allowing overexpression of ParAm in Tth & this study \\
\hline pMK-parBm & pMK18 derived vector, allowing overexpression of ParBm in Tth & this study \\
\hline pMK-parABm & pMK18 derived vector, allowing overexpression of ParABm in Tth & this study \\
\hline pET21a & expression vector, $\mathrm{P}_{\mathrm{T} 7}$, lacl, pBR322 ori, $\mathrm{Amp}^{\mathrm{R}}$ & Novagen, Germany \\
\hline pET21a-parBc & pET21a derived vector, allowing overexpression of ParBc in EC & this study \\
\hline pET21a-parBm & $\mathrm{pET} 21$ a derived vector, allowing overexpression of $\mathrm{ParBm}$ in $E C$ & this study \\
\hline pMK-sgfp & pMK18 derived vector, allowing expression of sGFP in Ec and Tth & this study \\
\hline pMKparBc-sgfp & pMK18 derived vector, allowing expression of parBc-sGFP in Ec and Tth & this study \\
\hline pMKparBm-sgfp & pMK18 derived vector, allowing expression of parBm-sGFP in Ec and Tth & this study \\
\hline T. thermophilus strains & Description & Source/reference \\
\hline HB27 & Thermus thermophilus & DSM 7039 \\
\hline $\mathrm{HB} 27 \Delta b g l$ & deletion of ORF TT_P0042 in HB27 & [69] \\
\hline TL-1 & carotenoid synthesis deficient, otherwise is considered as wild type & this study \\
\hline$\triangle p a r A B c$ & HB27 derivative with parABC replaced by kat & this study \\
\hline$\triangle p a r A m N-1$ & $\begin{array}{l}\text { HB27 derivative with the N-terminal region of parAm replaced by blm in parABm } \\
\text { transcription opposite direction }\end{array}$ & this study \\
\hline$\Delta$ parAmN-2 & $\begin{array}{l}\text { HB27 derivative with the N-terminal region of parAm replaced by blm in parABm } \\
\text { transcription co-linear direction }\end{array}$ & this study \\
\hline HB27/ParABm & HB27 derivative carrying a plasmid-borne copy of parABm & this study \\
\hline$\Delta p a r A m N-1 / P a r A B m$ & $\triangle p a r A m N-1$ derivative carrying a plasmid-borne copy of parABm & this study \\
\hline TMPO & HB27 derivative carrying pMK18 vector & this study \\
\hline TMP01 & HB27 derivative permitting overexpression of ParAm & this study \\
\hline TMP02 & HB27 derivative permitting overexpression of $\mathrm{ParBm}$ & this study \\
\hline TL-1/parBc-sGFP & TL-1 derivative permitting expression of ParBc-sGFP & this study \\
\hline TL-1/ParBm-sGFP & TL-1 derivative permitting expression of ParBm-sGFP & this study \\
\hline$\triangle p a r A m N-1 / P a r B m-s G F P$ & $\triangle$ parAmN-1 derivative permitting expression of ParBm-sGFP & this study \\
\hline
\end{tabular}

Tth, T. thermophilus; Ec, E. coli; Amp ${ }^{\mathrm{R}}$, ampicillin resistant; Blm${ }^{\mathrm{R}}$, bleomycin resistant; $\mathrm{Km}^{\mathrm{R}}$, kanamycin resistant; Tth (repA), replication origin for Tth; Ec (oriE), replication origin for $E c$; ori pUC, replication origin for pUC18.

qPCR was performed. The cDNA was synthesized from total RNA samples using the Maxima First Strand cDNA Synthesis Kit (Thermo Scientific, Germany). A chromosomally located constitutively expressed gene (TT_C1610) was chosen as an endogenous reference. The relative quantification method $\left(2^{-\Delta \Delta C}\right.$ T $)$ based on [65] was used in the calculations.

\section{Pulsed field gel electrophoresis (PFGE)}

PFGE was performed as described in [66], and the CHEF-DR ${ }^{\circ}$ III variable angle system was used for gel electrophoresis (Bio-Rad). $150 \mathrm{ml}$ 1\% PFGE certified agarose (Biozym Gold Agarose) prepared in $0.5 \times$ TBE was used for gel casting. The gels were run in $0.5 \times \mathrm{TBE}$ for $24 \mathrm{~h}$ under the following conditions: $6 \mathrm{~V} / \mathrm{cm}, 120 \mathrm{de}-$ gree included angle, $8-50$ sec switch time ramp, $14^{\circ} \mathrm{C}$.

\section{Purification of the ParBc and ParBm proteins}

ParB proteins were heterologously expressed in E. coli Rosetta 2 (DE3) after introduction of specifically constructed pET21a-based expression plasmids designated as pET21a-parBc and pET21a-parBm. Cultures were 
grown in $11 \mathrm{LB}$ medium (supplemented with chloramphenicol and ampicillin) at $37^{\circ} \mathrm{C}$. When the $\mathrm{OD}_{600}$ reached a value between 0.7 and 0.8 , protein expression was induced by the addition of IPTG at a final concentration of $1 \mathrm{mM}$ and the cultures were agitated at $30^{\circ} \mathrm{C}$ for $4 \mathrm{~h}$. The cells were harvested and lysed by sonication (UP200S, Hilscher, Teltow, Germany). After sonication, the crude cell extracts were centrifuged at $4{ }^{\circ} \mathrm{C}$ at $15,000 \mathrm{~g}$ for $30 \mathrm{~min}$ and the supernatants were subjected to affinity purification using Protino Ni-IDA 2000 columns (Macherey Nagel, Germany).

\section{Electrophoretic mobility shift assay (EMSA)}

For DNA binding assays, a 25-bp or a 18-bp DNA fragment that contained the chromosomal or megaplasmid parS sequence was used as the probe. The probes were 6-carboxyfluorescein (FAM)-labeled and were generated by hybridization of two complementary oligonucleotides. The chromosomal parS probe had the sequence:

5' -TGTTTCCCGTGAAACATCAGGCGCC-3' (WT parSc), and the megaplasmid pars probe had the sequence: 5'-GCAAGGACGCGTCCTTCA-3' (WT parSm). The binding reactions $(25 \mu \mathrm{l})$ were performed in $50 \mathrm{mM}$ $\mathrm{KCl}, 10 \mathrm{mM}$ Tris- $\mathrm{HCl}$ (pH 7.0), $1 \mathrm{mM}$ EDTA, $1 \mathrm{mM}$ DTT, $4 \%$ glycerol, $0.02 \mu \mathrm{g} / \mu \mathrm{l}$ Poly (dI-dC), and contained 15 pmol of FAM-labeled probe and varying amounts of ParB proteins. The reactions were incubated at $25^{\circ} \mathrm{C}$ for $30 \mathrm{~min}$ and then applied on $1 \%$ agarose gels prepared in $1 \times$ TBE buffer. The ParB-parS binding competition experiments were performed using both the unlabeled probes containing the wild-type parSc or the wild-type parSm sequence (wild-type competitor), and unlabeled probes that contained seven base-pair and eight base-pair changes in the parSc and parSm sites respectively (mutant competitor). The mutant parSc probe had the sequence: 5'-cGTgcCCaGgGAgACc TCAGGCGCC-3' (Mu parSc), and the mutant parSm probe had the sequence: 5'-GCtgtGtgcaGgCCTTCA-3' $(\mathrm{Mu}$ parSm). They were also generated by hybridization of two complementary oligonucleotides.

\section{Fluorescence microscopy}

For fluorescence microscopy, the cells from liquid cultures were collected by centrifugation $(5000 \mathrm{~g}, 10 \mathrm{~min})$, washed once with $1 \times$ PBS buffer and resuspended in the same volume of $1 \times$ PBS buffer. Staining was performed by the addition of DAPI (4',6-diamidino-2-phenylindole-dihydrochloride) with a final concentration of $0.2 \mu \mathrm{g} / \mathrm{ml}$, and if necessary, by the addition of $10 \mu \mathrm{g} / \mathrm{ml}$ 6-carboxyfluorescein (CFS), followed by incubation at RT for $20 \mathrm{~min}$. The residual dyes were washed off and the cells were resuspended in $1 \times$ PBS buffer. Fluorescence microscopy was performed with a Zeiss AxioImager M1 microscope using filter sets "DAPI" for
DAPI, "AF488" for CFS and for the sGFP fluorescence of strains that expressing ParB-sGFP, respectively. The micrographic images were taken with an AxioCam $\mathrm{MRm}$ camera and analyzed with the Image J $(\mathrm{NIH}$, USA) and AxioVision software (Carl Zeiss, Germany).

\section{$\beta$-glucosidase activity assay for $T$. thermophilus}

$\beta$-glucosidase activity was measured with exponentially growing cells as described in [67]. The enzyme assays were performed with three independently grown cultures.

\section{Additional files}

\begin{abstract}
Additional file 1: Figure S1. Predictions of the chromosomal and megaplasmid origin and terminus regions in $T$. thermophilus HB27. The GenSkew software (http://genskew.csb.univie.ac.at/) was used to compute the normal and cumulative GC skew for the chromosome and the megaplasmid. The windowsize and stepsize for the chromosomal sequence were both set to $1000 \mathrm{bp}$, and those for the megaplasmid sequence were both set to $100 \mathrm{bp}$. (A) Cumulative GC skew of the chromosomal sequence. The maximum indicating the chromosomal terminus position is at $558,731 \mathrm{bp}$, the minimum representing the chromosomal origin region position is at 1,524,671 bp. (B) Cumulative GC skew of the megaplasmid sequence. The maximum and minimum values are at the megaplasmid sequence positions 189, 545 bp and 71, $689 \mathrm{bp}$, indicating the positions of the megaplasmid terminus and origin regions, respectively.
\end{abstract}

Additional file 2: Figure S2. Genotype confirmation of the $p a r A B m$ and parBm mutants in T. thermophilus. (A) Genotype confirmation of the parABm mutants by PCR using genomic DNA as template and primers flanking the deleted region (primer pairs parm-F/parm-R). The in silico predicted sizes are $3.99 \mathrm{kbp}$ for the wild-type allele and $2.73 \mathrm{kbp}$ for the $\triangle$ parABm allele. (B) Genotype confirmation of the parBm mutants by PCR (primer pairs parm-F/parm-R-2). The predicted sizes for the PCR products are $3.39 \mathrm{kbp}$ for the wild type and $3.13 \mathrm{kbp}$ for the $\triangle$ parBm allele.

Additional file 3: Figure S3. In vitro DNA binding of ParBc to parSC, and of ParBm to parSm measured by gel mobility shift assays. All reactions were performed under the same condition as described in the Methods section. Shifted DNA species were labeled with "bound", free DNA species were labeled with "free DNA". (A) Gel shift assays were performed with 15 pmol FAM-labeled DNA probe containing the 16-bp parSc site (probe sequence: 5'-TGTTTCCCGTGAAACATCAGGCGCC-3'), and with various concentrations of ParBC. (B) Gel shift assays were performed with 15 pmol FAM-labeled DNA probe containing the predicted 14-bp parSm site (probe sequence: 5'- GCAAGGACGCGTCCTTCA-3') and with various concentrations of ParBm.

Additional file 4: Table S1. Primers used in this study.

\section{Competing interests}

The authors declare that they have no competing interests.

\section{Authors' contributions}

$H L, A A$ and $W L$ designed the experiments, $H L$ and AA performed the experiments; VTTP and BL contributed to the construction of strains; $\mathrm{HL}, \mathrm{AA}$ and $W L$ wrote the manuscript. All authors read and approved the final manuscript.

\section{Acknowledgments}

We thank Maria Übelacker and Beate Schumacher for providing excellent technical assistance.

This work was supported by the Bundesministerium für Bildung, Wissenschaft, Forschung und Technologie (BMBF) within the framework of the GenoMik (Genomforschung an Mikroorganismen) funding measure and by the German Research Foundation (DFG) and the Technische Universität München within the funding programme Open Access Publishing. 
Received: 4 December 2014 Accepted: 10 April 2015

Published online: 18 April 2015

\section{References}

1. Glaser P, Sharpe ME, Raether B, Perego M, Ohlsen K, Errington J. Dynamic, mitotic-like behavior of a bacterial protein required for accurate chromosome partitioning. Genes Dev. 1997;11:1160-8.

2. Gordon GS, Sitnikov D, Webb CD, Teleman A, Straight A, Losick R, et al. Chromosome and low copy plasmid segregation in E. coli: visual evidence for distinct mechanisms. Cell. 1997;90:1113-21.

3. Webb CD, Graumann PL, Kahana JA, Teleman AA, Silver PA, Losick R. Use of time-lapse microscopy to visualize rapid movement of the replication origin region of the chromosome during the cell cycle in Bacillus subtilis. Mol Microbiol. 1998;28:883-92.

4. Viollier PH, Thanbichler M, McGrath PT, West L, Meewan M, McAdams HH, et al. Rapid and sequential movement of individual chromosomal loci to specific subcellular locations during bacterial DNA replication. Proc Natl Acad Sci. 2004;101:9257-62.

5. Sharpe ME, Errington J. Upheaval in the bacterial nucleoid: an active chromosome segregation mechanism. Trends Genet. 1999;15:70-4.

6. Gerdes K, Møller-Jensen J, Ebersbach G, Kruse T, Nordström K. Bacterial mitotic machineries. Cell. 2004;116:359-66.

7. Leonard TA, Møller-Jensen J, Löwe J. Towards understanding the molecular basis of bacterial DNA segregation. Philos Trans R Soc Lond B Biol Sci. 2005;360:523-35.

8. Lemon KP, Grossman AD. Localization of bacterial DNA polymerase: evidence for a factory model of replication. Science. 1998;282:1516-9.

9. Lemon KP, Grossman AD. Movement of replicating DNA through a stationary replisome. Mol Cell. 2000;6:1321-30.

10. Dworkin J, Losick R. Does RNA polymerase help drive chromosome segregation in bacteria? Proc Natl Acad Sci. 2002;99:14089-94.

11. Kruse T, Blagoev B, Løbner-Olesen A, Wachi M, Sasaki K, Iwai N, et al. Actin homolog MreB and RNA polymerase interact and are both required for chromosome segregation in Escherichia coli. Genes Dev. 2006;20:113-24.

12. Defeu Soufo HJ, Graumann PL. Actin-like proteins MreB and Mbl from Bacillus subtilis are required for bipolar positioning of replication origins. Curr Biol. 2003;13:1916-20.

13. Kruse T, Møller-Jensen J, Løbner-Olesen A, Gerdes K. Dysfunctional MreB inhibits chromosome segregation in Escherichia coli. EMBO J. 2003;22:5283-92.

14. Gitai Z, Dye NA, Reisenauer A, Wachi M, Shapiro L. MreB actin-mediated segregation of a specific region of a bacterial chromosome. Cell. 2005;120:329-41.

15. Austin $\mathrm{S}$, Abeles A. Partition of unit-copy miniplasmids to daughter cells. II. The partition region of miniplasmid P1 encodes an essential protein and a centromere-like site at which it acts. J Mol Biol. 1983;169:373-87.

16. Li Y, Dabrazhynetskaya A, Youngren B, Austin S. The role of Par proteins in the active segregation of the P1 plasmid. Mol Microbiol. 2004;53:93-102.

17. Ebersbach G, Gerdes K. The double par locus of virulence factor pB171: DNA segregation is correlated with oscillation of ParA. Proc Natl Acad Sci. 2001;98:15078-83.

18. Møller-Jensen J, Jensen RB, Löwe J, Gerdes K. Prokaryotic DNA segregation by an actin-like filament. EMBO J. 2002;21:3119-27.

19. Barillà D, Rosenberg MF, Nobbmann U, Hayes F. Bacterial DNA segregation dynamics mediated by the polymerizing protein ParF. EMBO J. 2005;24:1453-64

20. Ringgaard S, van Zon J, Howard M, Gerdes K. Movement and equipositioning of plasmids by ParA filament disassembly. Proc Natl Acad Sci. 2009;106:19369-74.

21. Gerdes K, Møller-Jensen J, Bugge Jensen R. Plasmid and chromosome partitioning: surprises from phylogeny. Mol Microbiol. 2000;37:455-66.

22. Lin DC, Grossman AD. Identification and characterization of a bacterial chromosome partitioning site. Cell. 1998;92:675-85.

23. Breier AM, Grossman AD. Whole-genome analysis of the chromosome partitioning and sporulation protein Spo0J (ParB) reveals spreading and origin-distal sites on the Bacillus subtilis chromosome. Mol Microbiol. 2007;64:703-18.

24. Livny J, Yamaichi Y, Waldor MK. Distribution of centromere-like parS sites in bacteria: insights from comparative genomics. J Bacteriol. 2007;189:8693-703.

25. Gerdes K, Howard M, Szardenings F. Pushing and pulling in prokaryotic DNA segregation. Cell. 2010;141:927-42.
26. Reyes-Lamothe R, Nicolas E, Sherratt DJ. Chromosome replication and segregation in Bacteria. Annu Rev Genet. 2012;46:121-43.

27. Ireton $\mathrm{K}$, Gunther 4th NW, Grossman AD. spooJ is required for normal chromosome segregation as well as the initiation of sporulation in Bacillus subtilis. J Bacteriol. 1994;176:5320-9.

28. Lee PS, Lin DC, Moriya S, Grossman AD. Effects of the chromosome partitioning protein Spo0J (ParB) on oriC positioning and replication initiation in Bacillus subtilis. J Bacteriol. 2003;185:1326-37.

29. Lee PS, Grossman AD. The chromosome partitioning proteins Soj (ParA) and Spo0J (ParB) contribute to accurate chromosome partitioning, separation of replicated sister origins, and regulation of replication initiation in Bacillus subtilis. Mol Microbiol. 2006;60:853-69.

30. Mohl DA, Gober JW. Cell cycle-dependent polar localization of chromosome partitioning proteins in Caulobacter crescentus. Cell. 1997;88:675-84.

31. Mohl DA, Easter Jr J, Gober JW. The chromosome partitioning protein, ParB, is required for cytokinesis in Caulobacter crescentus. Mol Microbiol. 2001;42:741-55.

32. Fogel MA, Waldor MK. A dynamic, mitotic-like mechanism for bacterial chromosome segregation. Genes Dev. 2006;20:3269-82.

33. Saint-Dic D, Frushour BP, Kehrl JH, Kahng LS. A parA homolog selectively influences positioning of the large chromosome origin in Vibrio cholerae. J Bacteriol. 2006;188:5626-31.

34. Yamaichi Y, Fogel MA, Waldor MK. par genes and the pathology of chromosome loss in Vibrio cholerae. Proc Natl Acad Sci. 2007;104:630-5.

35. Dubarry N, Pasta F, Lane D. ParABS systems of the four replicons of Burkholderia cenocepacia: new chromosome centromeres confer partition specificity. J Bacteriol. 2006;188:1489-96.

36. Ohtani N, Tomita M, Itaya M. An extreme thermophile, Thermus thermophilus, is a polyploid bacterium. J Bacteriol. 2010;192:5499-505.

37. Hu B, Yang G, Zhao W, Zhang Y, Zhao J. MreB is important for cell shape but not for chromosome segregation of the filamentous cyanobacterium Anabaena sp. PCC 7120. Mol Microbiol. 2007;63:1640-52.

38. Schneider D, Fuhrmann E, Scholz I, Hess WR, Graumann PL. Fluorescence staining of live cyanobacterial cells suggest non-stringent chromosome segregation and absence of a connection between cytoplasmic and thylakoid membranes. BMC Cell Biol. 2007;8:39.

39. Henne A, Brüggemann $H$, Raasch $C$, Wiezer $A$, Hartsch $T$, Liesegang $H$, et al The genome sequence of the extreme thermophile Thermus thermophilus. Nat Biotechnol. 2004;22:547-53.

40. Leonard TA, Butler PJ, Löwe J. Structural analysis of the chromosome segregation protein Spo0J from Thermus thermophilus. Mol Microbiol. 2004;53:419-32.

41. Leonard TA, Butler PJ, Löwe J. Bacterial chromosome segregation: structure and DNA binding of the Soj dimer-a conserved biological switch. EMBO J. 2005;24:270-82.

42. Nardmann J, Messer W. Identification and characterization of the dnaA upstream region of Thermus thermophilus. Gene. 2000;261:299-303.

43. Li Y, Austin S. The P1 plasmid in action: time-lapse photomicroscopy reveals some unexpected aspects of plasmid partition. Plasmid. 2002;48:174-8.

44. Cava F, de Pedro MA, Blas-Galindo E, Waldo GS, Westblade LF, Berenguer J. Expression and use of superfolder green fluorescent protein at high temperatures in vivo: a tool to study extreme thermophile biology. Environ Microbiol. 2008;10:605-13.

45. Hellman LM, Fried MG. Electrophoretic mobility shift assay (EMSA) for detecting protein-nucleic acid interactions. Nat Protoc. 2007;2:1849-61.

46. Marston AL, Errington J. Dynamic movement of the ParA-like Soj protein of B. subtilis and its dual role in nucleoid organization and developmental regulation. Mol Cell. 1999;4:673-82.

47. Lewis RA, Bignell CR, Zeng W, Jones AC, Thomas CM. Chromosome loss from par mutants of Pseudomonas putida depends on growth medium and phase of growth. Microbiology. 2002;148:537-48.

48. Errington J, Murray H, Wu L. Diversity and redundancy in bacterial chromosome segregation mechanisms. Philos Trans R Soc Lond B Biol Sci. 2005:360:497-505.

49. Heidelberg JF, Eisen JA, Nelson WC, Clayton RA, Gwinn ML, Dodson RJ, et al. DNA sequence of both chromosomes of the cholera pathogen Vibrio cholerae. Nature. 2000;406:477-83.

50. Gruber S, Errington J. Recruitment of condensin to replication origin regions by ParB/Spo0J promotes chromosome segregation in B. subtilis. Cell. 2009;137:685-96.

51. Sullivan NL, Marquis KA, Rudner DZ. Recruitment of SMC by ParB-parS organizes the origin region and promotes efficient chromosome segregation. Cell. 2009;137:697-707. 
52. Minnen A, Attaiech L, Thon M, Gruber S, Veening JW. SMC is recruited to oriC by ParB and promotes chromosome segregation in Streptococcus pneumoniae. Mol Microbiol. 2011;81:676-88.

53. Yamaichi Y, Gerding MA, Davis BM, Waldor MK. Regulatory cross-talk links Vibrio cholerae chromosome II replication and segregation. PLoS Genet. 2011;7:e1002189.

54. Murray H, Errington J. Dynamic control of the DNA replication initiation protein DnaA by Soj/ParA. Cell. 2008;135:74-84

55. Kadoya R, Baek JH, Sarker A, Chattoraj DK. Participation of chromosome segregation protein ParAl of Vibrio cholerae in chromosome replication. J Bacteriol. 2011:193:1504-14.

56. Scholefield G, Errington J, Murray H. Soj/ParA stalls DNA replication by inhibiting helix formation of the initiator protein DnaA. EMBO J. 2012;31:1542-55

57. Kato J, Nishimura $Y$, Imamura R, Niki H, Hiraga S, Suzuki H. New topoisomerase essential for chromosome segregation in E. coli. Cell. 1990;63:393-404

58. Huang TW, Hsu CC, Yang HY, Chen CW. Topoisomerase IV is required for partitioning of circular chromosomes but not linear chromosomes in Streptomyces. Nucleic Acids Res. 2013;41:10403-13.

59. Yamaichi Y, Fogel MA, McLeod SM, Hui MP, Waldor MK. Distinct centromere-like parS sites on the two chromosomes of Vibrio spp. J Bacteriol. 2007;189:5314-24.

60. Tanaka T, Kawano N, Oshima T. Cloning of 3-isopropylmalate dehydrogenase gene of an extreme thermophile and partial purification of the gene product. J Biochem. 1981;89:677-82.

61. Gibson DG, Young L, Chuang RY, Venter JC, Hutchison 3rd CA, Smith HO Enzymatic assembly of DNA molecules up to several hundred kilobases. Nat Methods. 2009;6:343-5.

62. Brouns SJ, Wu H, Akerboom J, Turnbull AP, de Vos WM, van der Oost J. Engineering a selectable marker for hyperthermophiles. J Biol Chem. 2005:280:11422-31.

63. De Grado M, Castan P, Berenguer J. A high-transformation-efficiency cloning vector for Thermus thermophilus. Plasmid. 1999;42:241-5.

64. Breuert S, Allers T, Spohn G, Soppa J. Regulated polyploidy in halophilic Archaea. PLoS One. 2006;1:e92.

65. Livak KJ, Schmittgen TD. Analysis of relative gene expression data using real-time quantitative PCR and the 2(-Delta Delta C(T)). Method. 2001;25:402-8.

66. Herschleb J, Ananiev G, Schwartz DC. Pulsed-field gel electrophoresis. Nat Protoc. 2007:2:677-84

67. Ohta T. Glucosidase as a reporter for the gene expression studies in Thermus thermophilus and constitutive expression of DNA repair genes. Mutagenesis. 2006;21:255-60.

68. Yanisch-Perron C, Vieira J, Messing J. Improved M13 phage cloning vectors and host strains: nucleotide sequences of the M13mp18 and pUC19 vectors. Gene. 1985;33:103-19.

69. Angelov A, Li H, Geissler A, Leis B, Liebl W. Toxicity of indoxyl derivative accumulation in bacteria and its use as a new counterselection principle. Syst Appl Microbiol. 2013;36:585-92.

\section{Submit your next manuscript to BioMed Central and take full advantage of:}

- Convenient online submission

- Thorough peer review

- No space constraints or color figure charges

- Immediate publication on acceptance

- Inclusion in PubMed, CAS, Scopus and Google Scholar

- Research which is freely available for redistribution 